\title{
Análise do acolhimento a partir das relações na Atenção Básica no município do Rio de Janeiro
}

\section{Reception analysis based on relationships in Primary Care in the city of Rio de Janeiro \\ Análisis de recepción basado en relaciones em Atención Primaria em la ciudad de Río de Janeiro}

\author{
Tarciso Feijó da Silva ${ }^{1} \odot$, Helena Maria Scherlowski Leal David ${ }^{1} \odot$, Valéria Ferreira Romano ${ }^{2} \odot$ \\ ${ }^{1}$ Universidade do Estado do Rio de Janeiro, RJ, Brasil. \\ ${ }^{2}$ Universidade Federal do Rio de Janeiro, RJ, Brasil.
}

\section{Resumo}

Objetivo: Analisar o acolhimento a partir das relações entre os profissionais na Atenção Básica, destacando sua configuração e a posição dos diferentes atores de uma unidade de Atenção Básica em Saúde do município do Rio de Janeiro.Métodos: Estudo de natureza descritiva, com abordagem quantitativa e com participação de 37 profissionais, cuja análise foi desenvolvida pela lente da análise de redes sociais, que permitiu mapear as relações entre os atores a partir do acolhimento e representá-las graficamente. Resultados: A presença de todos os atores na rede pressupõe a existência de vínculos e alguma dinâmica de troca de recurso, conhecimento ou informação, além de movimentos disparados a partir do acolhimento na perspectiva da produção do cuidado. Por sua vez, a centralidade na rede de atores de nível superior, como médicos e enfermeiros e agentes comunitários de saúde ocupando a periferia, convergem para tradução de um cuidado com foco no modelo biomédico. Conclusões: Observou-se relevante papel do acolhimento ao ditar formas de organização e funcionamento, assim como por trazer relevo sobre a produção do cuidado existente no campo, que vai de encontro ao prescrito e institucionalizado nas políticas normativas e distanciase do acolhimento-ação necessário e esperado para a Atenção Básica em Saúde.

Palavras-chave: Atenção Primária à Saúde; Acolhimento; Redes Sociais; Assistência Centrada no Paciente.

\begin{abstract}
Objective: Analyze the reception based on the relationships between professionals in Primary Care, highlighting its configuration and the position of the different actors in a Primary Health Care unit in the city of Rio de Janeiro.Methods: A descriptive study with a quantitative approach involving 37 professionals, whose analysis was developed through the lens of Social Network Analysis, which allowed the mapping of relationships between the actors from the host and represent them graphically. Results: The presence of all actors in the network presupposes the existence of bonds and some dynamics of exchange of resources, knowledge or information, as well as movements triggered from the reception in the perspective of care production. In turn, the centrality in the network of higher level actors, such as doctors and nurses and community health agents occupying the periphery, converge to translate care focused on the biomedical model. Conclusions:It was observed an important role of the host in dictating forms of organization and operation, as well as bringing emphasis on the production of care in the field, which meets the prescribed and institutionalized in normative policies and away from the host-action necessary and expected for Primary Health Care.
\end{abstract}

Keywords: Primary Health Care; User Embracement ; Social Networking; Patient-Centered Care.

Como citar: Silva TF, David HMSL, Romano VF.Análise do acolhimento a partir das relações na Atenção Básica no município do Rio de Janeiro. Rev Bras Med Fam Comunidade. 2020;15(42):2326. https://doi.org/10.5712/rbmfc15(42)2326
Autor correspondente: Tarciso Feijó da Silva. E-mail: tarcisofeijo@yahoo.com.br Fonte de financiamento: declaram não haver. Parecer CEP: não encomendado* Procedência: externa Recebido em: 20/12/2019. Aprovado em: 17/05/2020. 


\section{Resumen}

Objetivo: Analizar la recepción em función de las relaciones entre profesionales de Atención Primaria, destacando su configuración y la posición de los diferentes actores en una unidad de Atención Primaria de Salud em la ciudad de Río de Janeiro. Método: Estudio descriptivo com un enfoque cuantitativo enel que participaron 37 profesionales, cuyo análisis se desarrolló a través de la lente del análisis de redes sociales, que permitió el mapeo de las relaciones entre los actores de la recepcióny representar lãs gráficamente. Resultados: La presencia de todos los actores em la red presupone la existencia de lazos y algunas dinámicas de intercambio de recursos, conocimiento o información, así como movimientos desencadenados desde la recepciónen la perspectiva de la producción asistencial. A su vez, la centralidade nla red de actores de nivel superior, como médicos y enfermeras y agentes de salud comunitarios que ocupan la periferia, convergen para traducir la atención centrada enel modelo biomédico. Conclusiones: Se observóun papel importante de la recepción em dictar formas de organización y operación, así como pon er énfasis en la producción de atención enel campo, que cumple com lo prescrito e institucionalizado em las políticas normativas y lejos de larecepción-acción necesaria y esperada para la Atención Primaria de Salud.

Palabras clave: Atención Primaria de Salud; Acogimiento; Red Social; Atención Dirigida al Paciente.

\section{INTRODUÇÃO}

O acolhimento no contexto da Atenção Básica em Saúde (ABS) é visto como estratégia para organização do processo de trabalho, gestão das demandas, melhoria do acesso e qualidade da atenção em saúde. ${ }^{1,2}$ Nesse ponto da rede de serviços de saúde espera-se um acolhimento conformado em rede a partir de relações sustentáveis, dinâmicas de troca de recursos eficientes e produção do cuidado com ênfase em atividades de vigilância em saúde..$^{2,3}$

AABS caracteriza-se pela orientação dos serviços que devem estar voltados para a comunidade;pela centralidade da atenção na família, bem como por sua capacidade de responder às necessidades de saúde de seus membros; e pela competência cultural para se comunicar e reconhecer as diferentes necessidades dos diversos grupos populacionais. ${ }^{4}$ Sua organização a partir do acolhimento exige que os profissionais reflitam sobre o conjunto de ofertas que a ABS tem apresentado para lidar com as necessidades de saúde da população e território, assim como que a equipe defina quais profissionais irão receber o usuário que chega; como serão avaliados os usuários em risco e vulnerabilidade; quais os fluxos e protocolos utilizados para encaminhamento; e como serão organizadas as agendas dos profissionais para o cuidado. ${ }^{5}$

As redes são consideradas decisivas, seja para organização do sistema de saúde, que implica em ações coordenadas de diversos serviços e setores, seja para constatação empírica de que o cotidiano e a manutenção da vida parecem apoiados em diferentes tipos de conexões (familiares, vizinhança, trabalho, amizade, etc.). ${ }^{6} \mathrm{Em}$ associação com o termo "social" a palavra rede tem sido utilizada para compreender as relações e a troca de recursos entre diferentes atores e como ferramenta para análise de processos. ${ }^{7}$

As redes sociais são construídas a partir de um capital social em constante movimento, constituídas a partir de relações vinculadas a um grupo de agentes que não são apenas dotados de propriedades comuns, mas unidos por ligações permanentes e úteis. ${ }^{8}$ Desta forma, presume-se que a ABS é um campo fértil para a existência de redes sociais, considerando as características do processo de trabalho nesse campo.

As redes sociais estão conectadas por relações/interações sociais que podem ser motivadas por amizade e por relações de trabalho ou compartilhamento de informações, onde, por meio dessas ligações, a estrutura social vai se (re)construindo. ${ }^{9}$ Assim, conjectura-se que as redes sociais instituídas a partir do acolhimento podem contribuir para uma ABS mais efetiva, mediada pelo trabalho multiprofissional, interdisciplinar, com foco na integralidade e equidade da atenção. Essas podem constituir-se ainda, como elementares para o conhecimento sobre a lógica de organização e funcionamento de determinado campo, 
assim como para conhecer o modelo de atenção em saúde que prevalece e que orienta a produção do cuidado.

Nesta perspectiva, o objetivo desse artigo é analisar o acolhimento a partir das relações entre os profissionais na Atenção Básica, destacando sua configuração e a posição dos diferentes atores de uma unidade de Atenção Básica em Saúde do Município do Rio de Janeiro.

\section{MÉTODOS}

Trata-se de estudo de natureza descritiva com abordagem quantitativa. A abordagem quantitativa pela lente da Análise de Redes Sociais (ARS) foi adotada por focar os padrões de relacionamento, ressaltar a objetividade das relações, possibilitar o mapeamento do fluxo da informação e os padrões de comunicação, e revelar os atores em posições de destaque na rede. ${ }^{10}$ Através dela, buscou-se não só medir os padrões de relacionamentos e as intercessões entre os atores, tendo como base seus contatos. Antes, com a utilização de procedimentos metodológicos, foi possível avançar mapeando as configurações sociais, representando os elos e as conexões existentes entre os diferentes atores na estrutura social. ${ }^{11}$

$O$ acolhimento e a ARS possuem como ponto de convergência a relação. O primeiro desponta na literatura como tecnologia relacional, ${ }^{12}$ já o segundo caracteriza-se por tomar a relação como objeto de análise. ${ }^{13}$ Dessa forma, a partir do acolhimento, buscou-se, apoiado na ARS, configurar o traçado das redes sociais dos atores e apresentar medidas que especificam os padrões de relacionamento entre os mesmos, o que, na pesquisa, mostrou-se relevante por evidenciar no contexto da ABS o papel de cada profissional, assim como esclarecer que atores são relevantes para o funcionamento do campo da ABS e quais recursos eles mobilizam para a produção do cuidado.

O cenário escolhido foi o Centro Municipal de Saúde (CMS) Parque Royal, que tem como área de abrangência o sub-bairro da Portuguesa, no bairro da llha do Governador (Comunidade do Parque Royal). A Comunidade do Parque Royal é composta, em sua maioria, por imigrantes da região Nordeste do Brasil, possuindo um total de 9.093 cidadãos cadastrados em prontuário eletrônico. ${ }^{14}$ Ressalta-se, entretanto, que todo o território do CMS Parque Royal é coberto por equipes de saúde da família, sendo assim denominado por não seguir o modelo previsto pela Casa Civil do Governo Federal, destoando das outras unidades no que concerne à estrutura física. ${ }^{15} \mathrm{~A}$ unidade pertence à Área Programática (AP)3.1, sendo uma das 03 Unidades de $A B$ existentes no território.

A estrutura da unidade é toda de alvenaria, sendo conformada por áreas de recepção, farmácia, saúde bucal, escovódromo, depósito de material e limpeza (DML), expurgo, esterilização, coleta, imunização, curativo, almoxarifado, salas de atendimento das equipes de saúde da família (sendo uma para cada equipe - Boa esperança, Emanuel e Tubiacanga), sala de saúde da mulher, corredor central com cadeiras dispostas próximo às diferentes salas de atendimento e ambiente aberto reservado (porém aberto) no final da unidade, com cerca de $10 \mathrm{~m}^{2}$ utilizado para realização de grupos de promoção da saúde. A recepção é apartada da unidade e conta com um guichê de atendimento por equipe de saúde da família. O profissional Agente Comunitário de Saúde (ACS) recebe os usuários que procuram os serviços ofertados pela unidade.

A escolha da unidade se deu pela possibilidade de inserção do pesquisador que já atuou como apoiador institucional na referida AP; pelo tempo de atuação da unidade no território que funciona há mais 
de 15 anos, o que pode contribuir para a vinculação dos usuários e o conhecimento deles dos serviços ofertados pela mesma; assim como pela quantidade de equipes existentes na unidade, já que as outras unidades possuíam número maior de equipes e o pesquisador tinha a pretensão de analisar as redes completas ou totais que os profissionais acionam a partir do acolhimento na perspectiva de produção do cuidado.

Participaram do estudo todos os profissionais que atuavam na unidade na ocasião da coleta de dados, independentemente da categoria profissional, do tipo de vínculo e da carga horária. O único critério utilizado para a inclusão dos mesmos foi está ativo no Cadastro Nacional de Estabelecimentos de Saúde (CNES) e estar vinculado à unidade no momento da coleta de dados. Nessa ordem, foram selecionados 37 profissionais, sendo: 01 gerente, 04 médicos, 03 enfermeiros, 01 dentista, 01 farmacêutico, 04 profissionais do Núcleo Ampliado de Saúde Família - Atenção Básica (NASF - AB), 03 técnicos de enfermagem, 01 auxiliar de saúde bucal (ASB), 13 agentes comunitários de saúde (ACS), 03 agentes de vigilância em saúde (AVS), 02 auxiliares administrativos e 01 auxiliar de serviços gerais.

A Equipe Emanuel é constituída por um médico, um enfermeiro, um técnico de enfermagem, um AVS e cinco ACS; a Boa Esperança por dois médicos, um enfermeiro, um técnico de enfermagem, um AVS e quatro ACS; já a Tubiacanga um médico, um enfermeiro, um técnico de enfermagem, um AVS e quatro ACS. Odentista e o ASB conformam a equipe de saúde bucal. O NASF - ABda unidade possui na sua composição o educador físico, o assistente social, o psicólogo e o fisioterapeuta. Por sua vez, o gerente, o farmacêutico, os auxiliares administrativos e o auxiliar de serviços gerais atuam integrados às equipes numa perspectiva de apoio.

Reitera-se que, durante o período de coleta de dados, não havia profissionais afastados por férias, licenças ou problemas de saúde e que, mesmo diante de um cenário de greve - aderida pelos profissionais médicos, enfermeiros, técnicos de enfermagem e ACS -, foi possível a abordagem e participação de todos os atores do campo.

A técnica utilizada para a coleta de dados foi a aplicação de um questionário on-line estruturado. Visando traçar e representar graficamente a rede social dos profissionaisacionadas pelo acolhimento com foco na produção do cuidado, além de informações que permitiram identificar o perfil dos profissionais (sexo, gênero, nível de escolaridade e tempo de atuação na unidade), uma única pergunta foi disparada pelo questionário on-line estruturado "Selecione todos os profissionais que você aciona a partir do acolhimento para a produção do cuidado". O acolhimento, no questionário, foi tipificado como uma tecnologia que envolve a relação, corroborando para deixar claro que outros atores os profissionais entrevistados consideravam relevantes para o atendimento das necessidades de saúde dos usuários. Uma relação nominal, com todos os atores do campo de pesquisa e respectivas funções, complementou o formulário, sendo que os participantes não tinham limitação quanto à quantidade de atores que poderiam assinalar como sendo relevantes.

O questionário, uma vez elaborado, foi exportado para a plataforma do Google Forms, que permite a edição de questionários e outros formulários de pesquisa. Foi solicitado ao gerente da unidade, considerando o conhecimento ampliado que ele possui sobre o perfil dos profissionais do campo, assim como por sua capacidade de respostas aos diferentes processos comunicativos instituídos no cotidiano do trabalho, que ele que validasse o instrumento visando verificar a clareza da linguagem utilizada e a tipologia das perguntas descritas. Após a validação, por esse ator, o instrumento foi enviado por e-mail e outras mídias 
digitais disponíveis (WhatsApp) para todos os 37 profissionais do campo acompanhado do Termo de Consentimento Livre e Esclarecido.

A abordagem quantitativa ocorreu pela representação gráfica da rede social, o sociograma - o retrato da relação e do agrupamento dos atores na estrutura social. Por meio desse, foi possível identificar todos os atores citados a partir das respostas ao questionário, trazendo relevo para os mais centrais e os mais periféricos na rede, o que permitiu evidenciar os diferentes papéis ocupados pelos profissionais, compreender e explorar as suas interações sociais numa perspectiva de produção do cuidado.

Os softwares UCINET e NETDRAW ${ }^{16,17}$ foram utilizados nessa etapa, sendo o primeiro para a análise das informações e criação dos sociogramas, e o segundo para a geração e apresentação dos mesmos em formato de imagens.

Os dados coletados pelo questionário estruturado on-line passaram por uma primeira análise, visando a identificação de falhas no processo de preenchimento, foram salvos após as correções necessárias e, em seguida, exportados para uma planilha no Microsoft Excel, onde, além de todas as informações que permitiram caracterizar o perfil dos profissionais, foi possível visualizar as respostas dos atores à pergunta disparadora que tinha por objetivo conhecer os profissionais que cada um aciona, a partir do acolhimento para a produção do cuidado.

A matriz no UCINET trabalha com uma lógica de variáveis dicotômicas, que, quando inseridas, contribuem para o conhecimento das relações existentes entre os diferentes atores da rede. O valor "0" é informado na matriz quando um ator não está em relação com outro; já o valor "1" indica presença de relação entre os atores. Dessa forma, visando à construção da matriz, as informações da planilha sobre as relações estabelecidas pelos atores a partir do acolhimento para a produção do cuidado foram analisadas e trabalhadas de forma isolada.

No Microsoft Excel, uma matriz foi construída, e todos os atores da pesquisa foram dispostos na vertical e na horizontal, onde foi possível relacionar as relações existentes entre eles. Em substituição às suas categorias profissionais, optou-se por codificações, onde cada ator recebeu um nome em referência à sua categoria profissional e equipe de saúde em que estava inserido. Na planilha, para cada ator citado por outro ator, inseriu-se valores "0" ou " 1 ", considerando as respostas negativas ou afirmativas à pergunta disparada na coleta de dados. Reitera-se que nenhum ator poderia citar a si mesmo, então foi inserido o valor "0" nas linhas correspondentes àquela em que os atores estavam pareados.

A planilha construída no Excel com as informações sobre as relações dos atores do campo, em formato de matriz, foi exportada para o software UCINET. Neste, considerando o " $n$ " de atores participantes, na opção "dimensão" tanto para linhas como para colunas, inseriu-se o valor "37", e, na opção "modo", foi marcado o item "simétrica", pelo fluxo de informação estabelecido entre os atores na pesquisa, tida como regular. Em seguida, criou-se uma pasta específica para download da matriz gerada pelo UCINET, visando a geração posterior do sociograma, que recebeu o nome de "Rede de Acolhimento Parque Royal".

A visualização gráfica das redes, por sua vez, foi realizada através da ferramenta NETDRAW, que já vem integrada ao UCINET. O ícone do NETDRAW do programa UCINET foi acionado, e a matriz, salva anteriormente com o nome "Rede de Acolhimento Parque Royal", foi aberta pela opção Open UCINET Network Dataset, que devolveu como resultado a representação gráfica da "Rede de Acolhimento Parque Royal" com os vínculos entre os atores e os respectivos fluxos da rede. Essa, por sua vez, foi analisada 
considerando as relações entre o conjunto de atores, a posição dos mesmos na rede e as políticas normativas que orientam a produção do cuidado no campo da ABS.

Por se tratar de um estudo que envolve seres humanos, exigências éticas e científicas fundamentais foram atendidas, sendo a pesquisa realizada de acordo com a Resolução n 466/2012 do Conselho Nacional de Saúde, que trata de pesquisas científicas envolvendo seres humanos. ${ }^{18} \mathrm{O}$ estudo teve anuência institucional da Secretaria Municipal de Saúde do Rio de Janeiro e aprovação do Comitê de Ética da Universidade do Estado do Rio de Janeiro, sob n 3.035 .782 , e Comitê de Ética da Secretaria Municipal de Saúde do Rio de Janeiro, sob n 3.103.928.

\section{RESULTADOS E DISCUSSÃO}

Nos últimos anos, o Brasil e, especificamente, o município do Rio de Janeiro vivenciaram tensões no cenário político e econômico associadas à corrupção e à segurança. Atrelado a isso, a edição da nova PNAB e a saída dos médicos cubanos do Programa Mais Médicos deflagraram rupturas e instabilidade no campo da ABS, afetando as relações dos atores e o processo de trabalho em diferentes contextos.

O CMS Parque Royal até a finalização da pesquisa não tinha sofrido impactos com o desligamento de profissionais, nem com a saída de médicos cubanos, já que todos os médicos lotados na ocasião da coleta de dados eram brasileiros. Evidenciou-se desmotivação por parte dos profissionais relacionada ao atraso salarial e às condições estruturais do campo. No entanto, esses problemas não foram identificados como capazes de alterar a dinâmica das relações existentes entre os atores e não influenciaram a participação deles na pesquisa, que demonstraram receptividade à mesma.

A maior parcela de profissionais $(81,1 \%)$ é do sexo e gênero feminino e a menor $(18,9 \%)$ é do sexo e gênero masculino. Com relação à variável nível de escolaridade, 16 (43,2\%) dos participantes do estudo possuem ensino superior completo, $19(51,4 \%)$ ensino médio completo, $01(2,7 \%)$ ensino fundamental completo e $01(2,7 \%)$ ensino fundamental incompleto. Do total de profissionais com nível superior, 05 $(13,5 \%)$ não possuem formação complementar, 05 (13,5\%) possuem especialização lato-sensu, 03 (8,1\%) especialização nos moldes de residência multiprofissional e 03 (8,1\%) mestrado acadêmico. Do total de profissionais, 67,6\% (25) atuam na unidade há mais de 03 anos, sendo a maior parte $13(35,2 \%)$ ACS.

$\mathrm{Na}$ ARS, a informação sobre o padrão dos laços entre os atores permite identificar os subgrupos reticulares com maior nível de coesão interna, assim como os papéis e as posições sociais manifestas pelas relações observadas entre os mesmos na rede. ${ }^{19}$ Cada participante citou todos os profissionais que aciona a partir do acolhimento para a produção do cuidado, sendo gerada a rede social denominada "Rede de produção do cuidado do CMS Parque Royal" (Gráfico 1).

O sociograma que emergiu retrata as relações dos profissionais no campo, sendo também o retrato do próprio campo, conformado pelos agentes que nele atuam. Ele expressa o momento presente e traz no seu bojo certa sazonalidade que permite apreender a perspectiva do trabalho dos atores, a relevância dos mesmos no campo da ABS e como a dinâmica das relações entre eles contribui para a produção do cuidado. Neste sentido, a rede como um todo, assim como as posições que os atores nela ocupam, não deve ser vista, nem analisada como estática, já que as mudanças associadas à entrada e saída de atores, assim como conflitos e crises, podem alterar a estrutura social e a dinâmica no campo da ABS, convergindo a qualquer tempo para sua reconfiguração. 


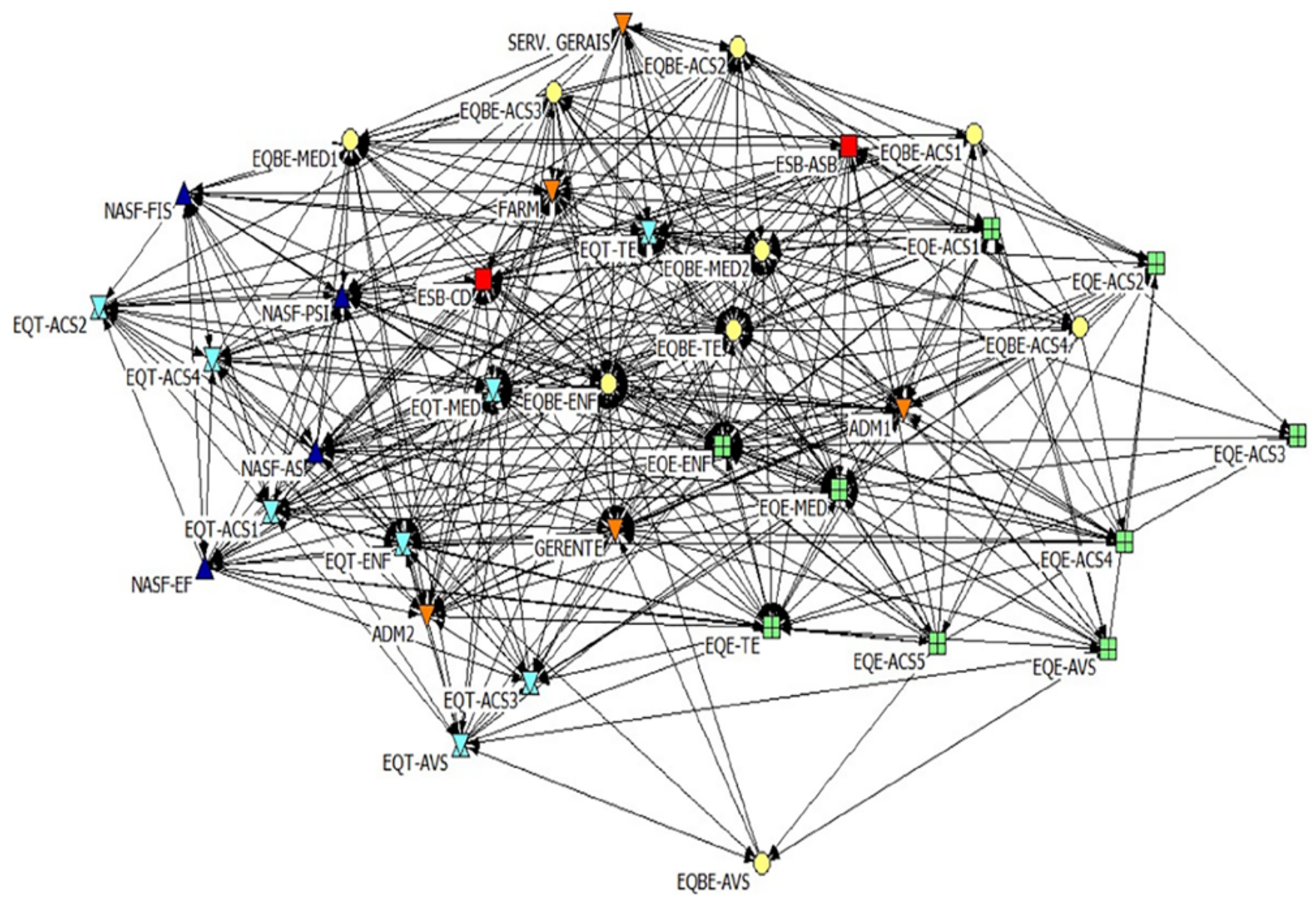

Gráfico 1. Rede de produção do cuidado do CMS Parque Royal. EQE: Equipe Emanuel; EQBE: Equipe Boa Esperança; EQT: Equipe Tubiacanga; ESB: Equipe Saúde Bucal; NASF: Núcleo Ampliado de Saúde da Família e Atenção Básica; Apoio e Gestão; MED: Médico; ENF: Enfermeiro; TE:Técnico de Enfermagem; AVS: Agente de Vigilância em Saúde; ACS: Agente Comunitário de Saúde; PSI: Psicólogo; EF: Educador Físico; AS: Assistente Social; FIS: Fisioterapeuta; FARM: Farmacêutico; ADM: Administrativo; SERV. GERAIS: Auxiliar de serviços gerais. Fonte: Os autores, 2019.

A "Rede de produção do cuidado do CMS Parque Royal" é do tipo completa, por envolver todos os atores de determinada organização e buscar evidenciar todos os tipos de vínculos que existe entre os mesmos; ${ }^{20}$ simétrica, pelo fluxo de comunicação instalado considerado como bidirecional; ${ }^{21}$ e, monomodal, por contemplar relações entre atores do mesmo tipo, no caso agentes do campo da saúde, inseridos em uma mesma estrutura social. ${ }^{16}$

$\mathrm{Na}$ rede os atores são representados por nós e as relações por traços, sendo possível identificar através do sociograma as três equipes de saúde da família, a equipe de saúde bucal, os atores da equipe NASF e os profissionais que atuam na perspectiva do apoio e da gestão no cenário do estudo. Esses, por sua vez, foram representados no diagrama de redes por códigos.

Para compreensão da estrutura social, é relevante reiterar que no cenário de pesquisa ocorrem processos de microgestão regulados por uma equipe de saúde da família. Assim,cada uma das equipes de saúde da família é responsável por uma população adscrita, tendo na sua composição 01 médico de 40 horas (Equipe Emanuel e Tubiacanga) ou 02 médicos de 20 horas (Equipe Boa Esperança), um enfermeiro, um técnico de enfermagem, de 04 a 06 agentes comunitários de saúde e um agente de vigilância em saúde. 
Os profissionais de nível superior das equipes são responsáveis pela supervisão das ações, educação permanente e avaliação das ações desenvolvidas pelos profissionais de nível médio, assim como de planejamento, desenvolvimento e análise dos resultados das atividades realizadas pela equipe; a única equipe de saúde bucal compartilha do processo de trabalho com as 03 equipes de saúde da família e responde por toda a população adscrita à unidade, sendo formada por uma auxiliar de saúde bucal e uma cirurgiã-dentista. Esta última atuando como gestora dos processos relacionados à odontologia.

O psicólogo, educador físico, fisioterapeuta e a assistente social integram a equipe NASF, tendo como objetivo contribuir para a integralidade do cuidado aos usuários do SUS, principalmente por meio da ampliação da clínica, auxiliando no aumento da capacidade de análise e de intervenção sobre problemas e necessidades de saúde, tanto em termos clínicos quanto sanitários, ${ }^{5}$ sendo que a liderança e o direcionamento desses profissionais e dos outros profissionais de nível superior e a gestão macro de todos os processos do campo competem à gerente da unidade, que delega para as duas administrativas que a assistem responsabilidades acerca de determinadas demandas. O campo possui ainda uma auxiliar de serviços gerais que atua em tempo integral.

Percebe-se a priori, através do sociograma,que todos os atores estão inseridos na rede, o que sinaliza que todos foram citados em algum momento por pelo menos 01 ator. $\mathrm{O}$ fato de todos os atores estarem na rede indica a existência de vínculos, dinâmica de troca de recurso, conhecimento ou informação e movimentos disparados pelos mesmos a partir do acolhimento na perspectiva da produção do cuidado. Não obstante, é relevante reiterar que a rede, na ABS, envolve análise do perfil populacional, programação da assistência considerando os riscos e as vulnerabilidades identificados e a utilização de diferentes tipos de tecnologias para alcance dos resultados esperados. ${ }^{22}$

As tecnologias podem ser classificadas como leve, leve-dura e dura. As tecnologias leves são as das relações; as leve-duras as dos saberes estruturados, tais como as teorias; e, as duras, as dos recursos materiais. A utilização da primeira no trabalho em saúde perpassa por processos de acolhimento, vínculo e atenção integral, como gerenciadores das ações de saúde. ${ }^{23}$

Para ser considerado como tecnologia leve, o acolhimento deve ocorrer para além da influência do contexto físico, onde o cuidado se dá, uma vez que um ambiente acolhedor se objetiva em um conjunto de ações, dentre elas na construção da relação com o outro. ${ }^{24}$ No grafo, é possível observar múltiplas relações disparadas pelo acolhimento, o que traz relevo sobre o mesmo como potente ferramenta para a comunicação entre os profissionais, vinculação dos usuários aos serviços, integralidade da atenção e produção do cuidado.

No cenário todos os profissionais atuam de forma permanente, 40 horas por semana, de segunda a sexta-feira, visando uma produção do cuidado, com foco na integralidade e na longitudinalidade, com exceção dos profissionais do NASF-AB, que cumprem metade da carga horária de 40 horas em outra unidade de saúde e de dois médicos que cumprem carga horária de 20 horas. Segundo Souza et al ${ }^{25}$ é por meio do estabelecimento das relações que o ator desenvolve afinidades humanas sustentáveis, assumindo atitudes que proporcionam decisões compartilhadas, aproximações não convencionais e convívios entre diferentes. Quanto mais próximos os atores estiverem uns dos outros e disponíveis para a troca e o diálogo, maior será a probabilidade de identificarem por quais ações no campo cada ator responde, como também quais as competências inerentes aos mesmos que são determinantes para a produção do cuidado. 
Nesta perspectiva, a distância geodésica na ARS considera para as redes direcionadas e não valoradas que o caminho mais eficiente conectando dois atores é aquele com a menor quantidade de passos, ou seja, a menor distância. ${ }^{26}$ Esta foi aplicada no estudo para descrever a coesão da rede social a partir da avaliação das distâncias que separam os atores, considerando a quantidade de passos que separam os mesmos; os caminhos mais eficientes de ligação entre dois ou mais atores; para explicitar movimentos de afastamento de atores na rede produzidos por relações hierarquizadas que valorizam o conhecimento de determinados atores, em detrimento de outros; e o isolamento proposital dos atores inseridos no campo, mas não envolvidos e comprometidos com as ações do mesmo (Tabela 1).

Os dados da Tabela 1 permitem concluir que a distância geodésica entre os atores é curta, tendo uma média de 1,7 passos. Os atores EQBE-AVS e EQE-ACS3 são os atores mais equidistantes, estando a 04 passos um do outro, sendo que o ator EQBE-AVS encontra-se distante da maioria dos outros atores na rede. Todos os profissionais de nível superior, com exceção dos que estão vinculados ao NASF (NASFPSI, NASF-ED, NASF-FIS e NASF-AS), figuram como aqueles capazes de circular pela rede com maior facilidade, em detrimento dos profissionais ACS, que por vezes precisam percorrer dois ou três passos para acessar outro ator na rede.

A posição de proximidade dos profissionais médicos e enfermeiros dos demais profissionais registra maior prestígio e autoridade dessas profissões em relação às outras profissões do campo. Por sua vez, o distanciamento dos profissionais ACS dos outros atores prediz uma transição no trabalho desses atores. Outrora, a atividade de educação em saúde objeto do trabalho desse profissional demandava articulação com outros atores, com a comunidade e com a rede de atenção em saúde. Atualmente, sua atuação tem se caracterizado como uma atividade meio, instrumentalizada e utilitarista, mais comprometida com objetivos imediatistas do que estruturantes de um novo modelo de atenção. ${ }^{27}$

Percebe-se com o achado que, no campo, as posições desses atores - médicos/enfermeiros e ACS - tendem a ser demarcadas pelos recursos que possuem e pela utilização que fazem dos mesmos. Por mais que os ACS tenham conhecimento pleno do território, por residirem e conhecerem a realidade a que estão sujeitos, os usuários adscritos, e por maior que seja o capital social que detêm derivado das relações permanentes, duradouras e de proximidade construídas de forma cotidiana com os usuários e a comunidade, o que prevalece, dentro da equipe, é o capital cultural dos médicos e enfermeiros ditado pela representação social de que estes possuem conhecimento, habilidades, informações e qualificações intelectuais capazes de responder às necessidades dos usuários. ${ }^{28}$

Na ocasião do estudo, a responsabilidade pelo planejamento, pela organização, direção, coordenação, execução e avaliação dos serviços de Enfermagem e Medicina, ora vista como responsabilidade técnica, recaía sobre os atores EQBE-ENF e EQE-MED. O primeiro, segundo a distância geodésica, está a um passo de 27 do total de 36 atores possíveis de serem conectados; já o segundo, a um passo de 24 atores. No entanto, não se pode afirmar que a função ocupada pelos mesmos no campo contribua para a ligação direta com outros profissionais, considerando que as distâncias geodésicas dos outros médicos e enfermeiros da rede estão bem próximas das deles.

Os atores EQE-TE, EQBE-TE e EQT-TE comunicam-se com facilidade com a maior parte dos atores. O ator EQE-TE mantém contato direto com 19 atores e está a 02 passos dos restantes; o ator EQBE-TE mantém contato direto com 23 atores e está a 02 passos dos restantes; e o ator EQT-TE mantém contato 


\begin{tabular}{|c|c|c|c|c|c|c|c|c|c|c|c|c|c|c|c|c|c|c|c|c|c|c|c|c|c|}
\hline 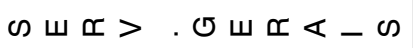 & $\sim$ & $\sim$ & - & $\sim$ & $\sim$ & $\sim$ & $\sim$ & $\sim$ & $\sim$ & $\sim$ & $\sim$ & $\sim$ & - & $m$ & - & - & - & N & $\sim$ & N & N & $N$ & N & - & $N$ \\
\hline$\varangle D \sum N$ & N & $\sim$ & - & $\sim$ & $\sim$ & $\sim$ & $\sim$ & $\sim$ & $N$ & - & - & - & - & $\sim$ & - & N & - & - & $\sim$ & - & $\sim$ & $N$ & $N$ & - & $\tau$ \\
\hline$\varangle D \Sigma-$ & $N$ & $\sim$ & - & - & - & $r$ & $\sim$ & - & N & N & $\sim$ & - & N & $\sim$ & - & - & - & - & $\sim$ & - & N & - & N & - & $\sim$ \\
\hline ৫ $ா \llbracket \mathbf{Z ~}$ & - & - & - & - & - & - & $\sim$ & - & N & $\sim$ & N & - & $\sim$ & - & - & - & - & - & - & $\sim$ & $\sim$ & - & $\sim$ & $\sim$ & N \\
\hline$\sqcup \varangle \simeq \Sigma$ & - & $N$ & $\sim$ & N & N & N & $\sim$ & $N$ & N & - & - & - & N & $\sim$ & - & - & - & N & - & - & N & $\sim$ & - & - & $\sim$ \\
\hline$Z \varangle \omega \sqcup, \sqcup-\omega$ & $\sim$ & N & $\sim$ & N & $\sim$ & m & $m$ & N & $N$ & - & - & - & $N$ & $\sim$ & $N$ & $N$ & N & N & - & - & N & $\sim$ & - & $\sim$ & N \\
\hline$Z \varangle \omega \sqcup, \varangle \omega$ & $\sim$ & N & $\sim$ & N & $\sim$ & $\sim$ & $m$ & $\sim$ & N & $\sim$ & - & - & - & $\sim$ & $\sim$ & - & $\sim$ & $\sim$ & - & - & $\sim$ & $N$ & $\sim$ & $\sim$ & - \\
\hline$Z \varangle \omega \sqcup, ш \amalg$ & $\sim$ & $N$ & $\sim$ & $m$ & N & m & $m$ & N & $\sim$ & $\sim$ & $\sim$ & - & N & $\sim$ & - & N & $\sim$ & $\sim$ & - & - & N & $\sim$ & $N$ & N & $\sim$ \\
\hline$Z \varangle \omega \sqcup, \square \omega-$ & N & $\sim$ & N & N & $\sim$ & N & の & N & $N$ & - & - & - & - & N & - & $N$ & - & $\sim$ & - & - & N & - & $\sim$ & - & $N$ \\
\hline Шのற, $の \oplus$ & N & $\sim$ & $\sim$ & $\sim$ & - & - & $m$ & - & - & $m$ & N & $\sim$ & $\sim$ & $\infty$ & m & $\sim$ & - & - & - & - & $\sim$ & N & $\sim$ & $\sim$ & N \\
\hline Шの®, ல & - & $N$ & $\sim$ & N & - & $\sim$ & $\sim$ & N & - & $\sim$ & - & - & - & $\sim$ & $\sim$ & N & - & N & - & - & N & N & - & - & N \\
\hline ш & N & $r$ & N & N & $\sim$ & N & $m$ & N & N & $\sim$ & N & $\sim$ & N & $\sim$ & $N$ & $N$ & N & N & - & - & - & - & $N$ & - & $N$ \\
\hline ш $0 \vdash, \varangle 0 \omega \mathrm{n}$ & N & $N$ & - & N & N & $\sim$ & $\sim$ & N & $N$ & $\sim$ & - & $\sim$ & - & $\sim$ & N & $\sim$ & $\sim$ & - & $\sim$ & $\sim$ & - & - & $N$ & $\sim$ & 0 \\
\hline ш & N & N & $\sim$ & $m$ & $\sim$ & N & $m$ & $N$ & $\sim$ & $\sim$ & N & N & N & $m$ & $N$ & $N$ & $\sim$ & $m$ & - & - & - & N & $N$ & 0 & $N$ \\
\hline ш & $\sim$ & N & $\sim$ & - & N & N & $m$ & $\sim$ & $\sim$ & $\sim$ & N & $\sim$ & N & $\sim$ & $N$ & $N$ & $\sim$ & N & - & - & - & - & 0 & - & N \\
\hline ш $\sigma \vdash, \varangle>\omega$ & $N$ & $N$ & $\sim$ & N & $\sim$ & $m$ & $m$ & $N$ & $\sim$ & の & $m$ & $\sim$ & $\sim$ & - & $N$ & $N$ & ल & $m$ & - & - & N & 0 & N & $\sim$ & $\sim$ \\
\hline ш & - & - & N & N & - & - & $\sim$ & N & $r$ & $\sim$ & - & $\sim$ & $\sim$ & ल & - & $\sim$ & - & N & - & - & 0 & N & - & - & - \\
\hline ш & $\sim$ & $N$ & - & N & N & $\sim$ & $\sim$ & - & $\sim$ & $\sim$ & $\sim$ & $\sim$ & - & $\sim$ & $N$ & N & $\sim$ & N & - & 0 & - & - & - & - & - \\
\hline ш & - & - & - & N & - & N & $\sim$ & $\sim$ & - & $\sim$ & N & - & - & N & N & - & $\sim$ & $\sim$ & 0 & - & $\sim$ & $\sim$ & - & - & - \\
\hline ш $\bigcirc \sqcap ш, \varangle \circlearrowright \omega \nabla$ & N & $N$ & $\sim$ & N & N & N & $m$ & N & $\sim$ & $\sim$ & - & $\sim$ & - & (n) & $N$ & - & $\sim$ & 0 & $\sim$ & $\sim$ & - & m & $N$ & $\sim$ & N \\
\hline ш & N & N & $\sim$ & N & $\sim$ & N & $m$ & $m$ & $\sim$ & N & - & $\sim$ & $\sim$ & N & $N$ & N & 0 & - & $\sim$ & N & - & - & $N$ & $\sim$ & N \\
\hline ш & N & N & $\sim$ & N & N & N & $m$ & $\sim$ & N & - & N & $\sim$ & - & N & $N$ & 0 & $\sim$ & $r$ & $\sim$ & $\sim$ & - & N & $N$ & $\sim$ & $N$ \\
\hline ш & $\sim$ & N & $m$ & $m$ & $\sim$ & N & $m$ & $m$ & - & - & N & $\sim$ & $\sim$ & $m$ & 0 & $\sim$ & $\sim$ & - & $\sim$ & N & - & $\sim$ & N & $\sim$ & $N$ \\
\hline$ш \bigcirc \sqcap ш, \varangle>\omega$ & $m$ & $m$ & $m$ & - & m & $m$ & $\nabla$ & $m$ & - & m & N & $\sim$ & N & 0 & $\sim$ & $N$ & m & N & $\sim$ & N & $\sim$ & - & $m$ & m & N \\
\hline ш & - & - & $\sim$ & - & - & - & N & - & - & - & - & $\sim$ & 0 & $\sim$ & - & - & - & - & $\sim$ & $\sim$ & $\sim$ & $\sim$ & - & $\sim$ & - \\
\hline ш ○ ш , ш & - & - & - & N & - & N & $\sim$ & - & - & - & - & 0 & - & 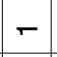 & - & - & - & - & $\sim$ & N & N & - & - & $\sim$ & - \\
\hline ш & - & - & - & - & - & N & $\sim$ & $\sim$ & - & $\sim$ & 0 & - & - & N & - & 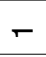 & - & - & $\sim$ & $\sim$ & $\sim$ & N & - & $\sim$ & - \\
\hline ш & $\sim$ & $r$ & N & N & - & N & $\sim$ & $N$ & $N$ & 0 & N & - & - & N & - & - & - & N & $\sim$ & N & $N$ & - & - & $\sim$ & - \\
\hline ш Ош, & N & N & $\infty$ & $m$ & $\sim$ & N & m & $m$ & 0 & $m$ & N & $\sim$ & N & $m$ & $\sim$ & $N$ & $\sim$ & $r$ & $\sim$ & $\sim$ & - & $m$ & $\sim$ & $\sim$ & $N$ \\
\hline ш & N & N & - & N & $\sim$ & N & $\sim$ & ○ & $\sim$ & $\sim$ & $\sim$ & $\sim$ & $\sim$ & $\infty$ & $\sim$ & $N$ & $\sim$ & - & $\sim$ & $N$ & - & N & $N$ & N & N \\
\hline ш О ш, «லの & N & N & - & $\sim$ & $\sim$ & N & 0 & $m$ & - & $\sim$ & N & $\sim$ & $m$ & $\infty$ & - & $m$ & $\sim$ & $\sim$ & $\sim$ & $N$ & - & N & N & N & $N$ \\
\hline ш & N & $N$ & - & - & $\sim$ & 0 & $\sim$ & - & - & N & N & $\sim$ & $\sim$ & $\sim$ & $\sim$ & - & $\sim$ & - & $\sim$ & $\sim$ & - & N & $N$ & $\sim$ & $\sim$ \\
\hline ш Ш , «லの - & N & N & - & - & 0 & N & $\sim$ & - & N & N & N & $\sim$ & N & $\sim$ & $N$ & - & N & N & $\sim$ & N & - & N & $N$ & N & $N$ \\
\hline ш б & $\sim$ & N & N & 0 & $\sim$ & N & $m$ & $N$ & $\sim$ & N & $m$ & $\sim$ & N & - & - & - & $N$ & $\sim$ & $N$ & $\sim$ & $N$ & - & $m$ & $m$ & $m$ \\
\hline ш & - & - & 0 & - & - & - & - & N & - & $\sim$ & - & - & $\sim$ & $\sim$ & $N$ & $N$ & N & $\sim$ & $\sim$ & N & N & - & - & $\sim$ & - \\
\hline ш ○ ш , ш Z ᄂ & - & ○ & - & - & - & - & - & - & - & $\sim$ & N & $\sim$ & - & $\sim$ & $N$ & $N$ & - & - & $\sim$ & N & $\sim$ & - & - & $\sim$ & - \\
\hline ه & 0 & - & - & - & - & - & - & $r$ & - & $\sim$ & - & - & - & $\sim$ & $N$ & $\sim$ & - & N & $\sim$ & N & - & N & - & N & - \\
\hline & 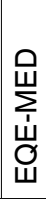 & 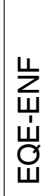 & 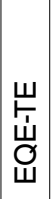 & 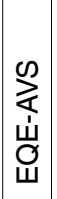 & 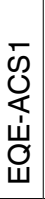 & 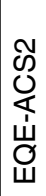 & 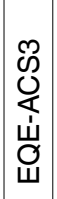 & 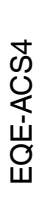 & 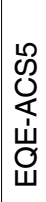 & 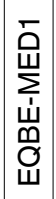 & 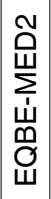 & $\begin{array}{l}\text { u } \\
\text { ய } \\
\text { ய் } \\
\text { ơ } \\
\text { ய }\end{array}$ & 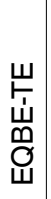 & 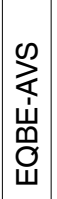 & 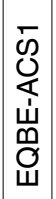 & 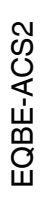 & 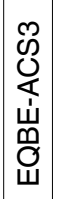 & 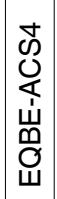 & 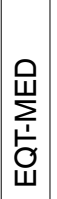 & 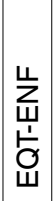 & 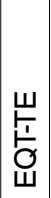 & 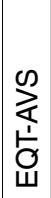 & 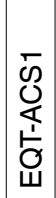 & 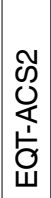 & 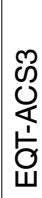 \\
\hline
\end{tabular}




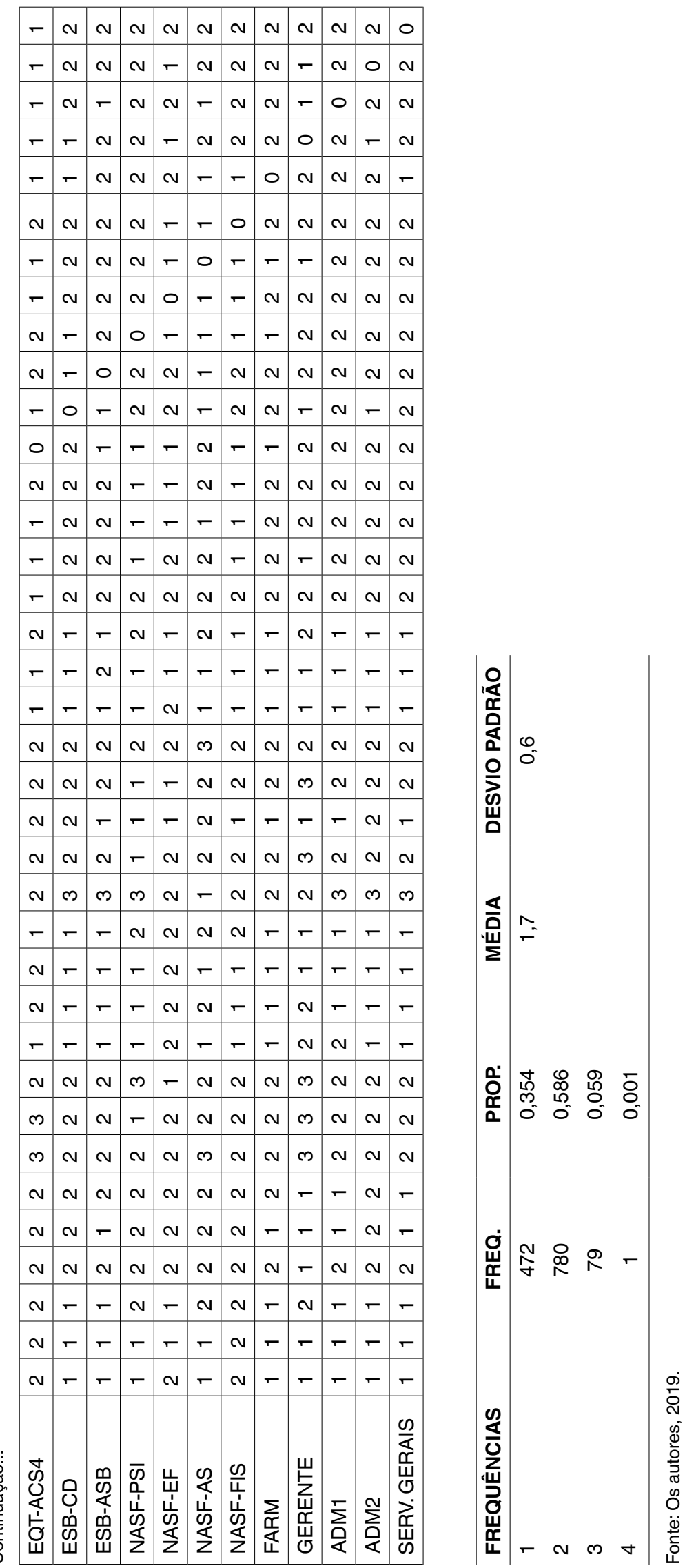


direto com 21 atores, estando a 02 passos de 14 atores e a 03 passos do ator EQBE-AVS. A distância geodésica desses profissionais, talvez, justifique-se pela atuação dos mesmos na escuta qualificada e no acolhimento da demanda espontânea, no direcionamento das mesmas para os profissionais de nível superior e pelo desenvolvimento dos principais procedimentos de cunho técnico. As atividades que os mesmos detêm na maior parte das vezes são disparadoras de outras ações que exigem a atuação de outros profissionais com maior capacidade resolutiva, haja vista a produção do cuidado esperada para cada caso.

Na ABS a participação no acolhimento dos usuários é vista como atividade que envolve o coletivo de profissionais que devem proporcionar um atendimento humanizado, realizando classificação de risco, identificando as necessidades de intervenções de cuidado, responsabilizando-se pela continuidade da atenção e viabilizando o estabelecimento do vínculo. ${ }^{29}$ Pelo fato do enfermeiro realizar e/ou supervisionar o acolhimento, assim como a classificação de risco, de acordo com protocolos previamente estabelecidos, observa-se, em determinados cenários, maior atuação desse ator. ${ }^{30}$ Quanto à participação do profissional técnico de enfermagem no acolhimento é mister esclarecer que a PNAB não descreve com clareza como deve ser delineada a sua participação, ${ }^{5}$ o que pode contribuir para que essas sejam orientadas à moda da gestão, implicando na produção do cuidado dos usuários, caso estes profissionais não tenham habilidades e conhecimentos para lidar com as diferentes situações identificadas a partir do acolhimento.

$\mathrm{Na}$ cadeia de atendimento que envolve o usuário no cenário de estudo, identifica-se prescrito, a presença de uma hierarquia que pode se configurar como elemento capaz de gerar sobrecarga sobre determinados profissionais em detrimento de outros. O enfermeiro emerge como principal responsável pelo acolhimento, podendo, em alguns momentos, ser substituído pelo profissional técnico de enfermagem - o que pode contribuir para a sobrecarga de trabalho desse último; fragilizar o papel e a relação desses profissionais; e a compreensão dos usuários acerca das ações esperadas para cada profissão; - o médico, por sua vez, na hierarquia instituída, constitui-se como elemento último da retaguarda, sendo acionado em situações críticas e para fins prescritivos, o que traz relevo sobre suas ações no campo. ${ }^{14}$ No entanto, a "Rede de produção do cuidado do CMS Parque Royal" gerada retrata certa divergência entre o prescrito e o esperado para o campo, já que no centro da rede e por sua vez envolvidos diretamente com o acolhimento aparecem atores de diferentes categorias, corroborando para a percepção de alguma tipologia de trabalho em equipe entre os mesmos.

A literatura nacional apresenta diferentes configurações para o acolhimento. ${ }^{29}$ Essas, mesmo que distintas, tomam o acolhimento como dispositivo para regulação do acesso e atendimento às demandas espontâneas e apesar de serem pautadas na escuta e na classificação de risco/vulnerabilidade,reiteram uma lógica de produção do cuidado verticalizada e baseada na relação queixa-conduta.

$\mathrm{Na} A B S$, o acolhimento deve transcender, sendo utilizado como uma estratégia de vigilância em saúde para a produção do cuidado. ${ }^{2}$ Essa modalidade de acolhimento envolve trazer para o centro da rede a maioria dos profissionais ACS e AVS, que no sociograma encontram-se na periferia e que são essenciais para criação de vínculo, levantamento das necessidades de saúde, integralidade da atenção, diagnóstico da situação de saúde e planejamento com base na realidade local. Tornar estes atores centrais na rede perpassa pela compreensão da relevância dos mesmos para a produção do cuidado pelos atores responsáveis, por direcionar as ações do campo e orientar o processo de trabalho (gerente, médicos 
e enfermeiros), que nesse ponto da rede deveria ir de encontro à vigilância em saúde e englobar um atendimento que tem como foco o indivíduo, a família e a comunidade.

A proposta de acolhimento como estratégia de vigilância em saúde para produção do cuidado deve orientar-se pela relação e envolver ação (acolhimento-ação); deve ser permeada por sensações e impressões, e não apenas pela escuta; considerar a atuação coletiva dos profissionais; ocorrer em todos os espaços e momentos do serviço, não devendo limitar-se ao recebimento de demanda espontânea, a identificação de risco ou definição de urgências; e explorar, no sentido de tornar útil, todos os recursos do campo, assim como o conhecimento dos profissionais. Essa nova modelagem que emergiu através da análise da Rede de produção do cuidado do CMS Parque Royal pressupõe envolvimento e responsabilização de todos os profissionais; conhecimento pleno da relação de serviços que o campo é capaz de ofertar aos usuários e aqueles passíveis de serem implementados a partir da realidade social e perfil epidemiológico da população; identificação dos recursos que cada profissional que atua no campo detêm e que podem ser úteis no atendimento das necessidades dos usuários; e, principalmente, atuação dos profissionais em rede, de forma colaborativa, tendo como atividade-fim a produção do cuidado.

O acolhimento-ação, enquanto práxis profissional na ABS deve estabelecer pontos de convergência com o paradigma indiciário proposto por Carlo Ginzburg e que tem sua raiz na semiótica médica. Este envolve um conjunto de princípios e procedimentos permeado pelo detalhe, pela análise dos dados marginais, dos resíduos tomados enquanto pistas, indícios, sinais e vestígios. ${ }^{31}$ Nessa ótica, o acolhimento-ação, pela sua capacidade de, a partir de dados aparentemente negligenciáveis, permitir compreender uma realidade complexa que não está em evidência à primeira vista; colocar em destaque particularidades e detalhes que podem contribuir para uma abordagem minuciosa e atenção a fatos aparentemente insignificantes; perceber que o pragmatismo e o atendimento protocolar podem ser elementos que impulsionam um atendimento ordenado, que não leva em conta a singularidade dos sujeitos; considerar que elementos imponderáveis como o faro, o golpe de vista e a intuição, o sentir e o perceber, quando presentes, pode municiar o profissional de saúde, tornando-o mais assertivo; fazendo-o valorizar as mudanças de comportamento, os sinais e pormenores pouco explorados, seguindo as pegadas deixadas pelos sujeitos acolhidos e que podem ser determinantes para a produção do cuidado.

Na prática dos serviços na ABS, o acolhimento-ação emerge como um desafio, já que o acolhimento dos usuários nos serviços de saúde ao longo dos anos foi sendo normatizado e diferentes documentos contribuíram para que houvesse formas oficiais de gestão do mesmo no contexto da ABS. Elas ora o tomam como um conhecimento estruturado para a organização da porta de entrada e do processo de trabalho, ora como uma tecnologia que envolve relação.

Guimarães $^{32}$ esclarece que o olhar, a escuta e o toque de um profissional de saúde experiente e envolvido com os problemas trazidos por seus pacientes são fundamentais para o bom julgamento clínico. Com a experiência, o profissional de saúde sabe distinguir um caso grave de uma cena corriqueira. Desse modo, vemos que a intuição tem pelo menos duas pernas, uma que está ancorada no conhecimento anterior ou já dado, e outra que advém no momento presente em que está se dando. Assim, por mais que apostemos no valor do acontecimento e do devir, não deixamos de acentuar o valor da memória e do conhecimento acumulado. Desta forma, o acolhimento-ação utiliza-se dessa memória e do conhecimento acumulado na 
perspectiva de ampliar o olhar sobre o sujeito, conhecendo suas demandas, porém avançando em direção às suas necessidades de cuidado.

No campo da $A B S$, diferentes capitais $^{33}$ estão em jogo, o que pode contribuir para que a ressignificação do acolhimento ora prescrito em um acolhimento-ação, pela orientação normativa tão presente na prática profissional, encontre resistência. O capital social permeia a prática dos ACS, que, em contato com os núcleos familiares, podem ampliar o olhar para questões que avançam para além do biológico, contribuindo para que esses tenham um sentimento de pertencimento ao campo da ABS; já o capital cultural se manifesta no campo através dos títulos escolares que os atores possuem, sendo que, pelo reconhecimento que os profissionais de nível superior possuem, eles tendem a manter domínio sobre as ações, diferente dos profissionais de nível médio, que, mesmo tendo formação diferenciada, pelo vínculo que assumem no campo, acabam por não terem o mesmo poder de fala e de tomada de decisão, implicando que ações assertivas ora propostas pelos mesmos não tenham a devida valorização, assim como os direcionamentos necessários e que poderiam ser relevantes para a produção do cuidado.

\section{CONCLUSÕES}

A "Rede de produção do cuidado do CMS Parque Royal" possibilitou identificar de que forma as relações a partir do acolhimento são construídas numa perspectiva de produção do cuidado, assim como qual a posição e o papel dos diferentes profissionais na rede.

A presença de profissionais de nível superior, como médicos e enfermeiros, centrais na rede e de ACS, ocupando posições periféricas, caracteriza formas de gerir o cuidado, cujo foco baseia-se no modelo biomédico, prescritivo e curativo, quando são esperadas para este campo ações voltadas para promoção e prevenção em saúde.

No estudo observou-se relevante papel do acolhimento ao ditar formas de organização e funcionamento, assim como por trazer relevo sobre a produção do cuidado existente no campo, que vai de encontro ao prescrito e institucionalizado nas políticas normativas e distancia-se do acolhimento-ação necessário e esperado para a ABS.

Não obstante, é necessário considerar que a saúde no Rio de Janeiro vem enfrentando nos últimos anos uma crise política, ética e estética. A mídia tem noticiado o descaso, o abandono e as dificuldades enfrentadas pelos diferentes serviços da rede de atenção para manterem suas atividades em pleno funcionamento e para gerir o cuidado em saúde.

A ABS como principal porta de entrada da rede de atenção em saúde tem sido uma das mais afetadas com atrasos salariais, falta de insumos e de apoio diagnóstico. No entanto, mesmo diante de um vazio assistencial instalado, percebe-se a relevância das redes sociais para lidar com as crises, seja internamente, através do coletivo que envolve uma única unidade de saúde, seja através da formação de redes de militância por parte de profissionais de diferentes unidades de saúde,que articulados e com apoio dos seus respectivos conselhos de classe e sindicatos podem de forma ampliada buscar e construir estratégias para lidar com as intempéries, na perspectiva de manterem suas atividades e, por conseguinte, o cuidado em saúde. 


\section{REFERÊNCIAS}

1. Silva TF, Romano VF. Sobre o acolhimento: discurso e prática em Unidades Básicas de Saúde do município do Rio de Janeiro. Saúde Debate. 2015 Jun;39(105):363-74. DOI: http://dx.doi.org/10.1590/0103-110420151050002005 DOI: https://doi.org/10.1590/0103110420151050002005

2. Silva TF, David HMSL, Caldas CP, Martins EL, Ferreira SR. O acolhimento como estratégia de vigilância em saúde para produção do cuidado: uma reflexão epistemológica. Saúde Debate. 2018 Dez;42(spe4):249-60. DOI:http://dx.doi.org/10.1590/0103-11042018s420 DOI: https://doi.org/10.1590/0103-11042018s420

3. Silva CT, Silva S, Almeida MV, Araújo I. Integralidade e suas interfaces com a produção do cuidado. RevCuid. 2014Jul/Dez;5(2):731-8. DOI: http://dx.doi.org/10.15649/cuidarte.v5i2.85 DOI: https://doi.org/10.15649/cuidarte.v5i2.85

4. Starfield B. Atenção Primária: equilíbrio entre necessidades de saúde, serviços e tecnologia. Brasília (DF): UNESCO/Ministério da Saúde; 2002.

5. Ministério da Saúde (BR). Portaria nํ⒉436, de 21 de setembro de 2017. Aprova a Política Nacional de Atenção Básica, estabelecendo a revisão de diretrizes para a organização da Atenção Básica, no âmbito do Sistema Único de Saúde (SUS). Diário Oficial da União, Brasília (DF), 21 set 2017: Seção 1.

6. Mendes EV. As redes de atenção à saúde. Brasília (DF): Organização Pan-Americana da Saúde (OPAS); 2011.

7. Hall A, Wellman B. Social network and social support. In: Cohen S, Syme SL, orgs. Social support and health. London: Academic Press; 1985. p. 23-41.

8. Andrade DMC, David HMSL. Análise de redes sociais: uma proposta metodológica para a pesquisa em saúde e na enfermagem. Rev Enferm UERJ. 2015Nov/Dez;23(6):852-65. DOI: http://dx.doi.org/10.12957/reuerj.2015.14861 DOI: https://doi.org/10.12957/ reuerj.2015.14861

9.Tomaél MI, Marteleto RM. Redes sociais: posições dos atores no fluxo da informação. Enc. Bibli: R. Eletr. Bibliotecon. Ci. Inf. 2007;(esp):7591. DOI: https://doi.org/10.5007/1518-2924.2006v11nesp1p75 DOI: https://doi.org/10.5007/1518-2924.2006v11nesp1p75

10. Marteleto RM. Análise de redes sociais: aplicação nos estudos de transferência da informação. Ci. Inf. 2001 Jan/Abr;30(1):71-81. DOI: http://dx.doi.org/10.1590/S0100-19652001000100009 DOI: https://doi.org/10.1590/S0100-19652001000100009

11. Marteleto RM, Tomaél MI. A metodologia de análise de redes sociais (ARS). In: Valentin MLP, org. Métodos qualitativos de pesquisa em Ciência da Informação. São Paulo: Polis; 2005. p. 81-100.

12. Teixeira RR. O acolhimento num serviço de saúde entendido como uma rede de conversações. In: Pinheiro R, Mattos RA, org. Construção da integralidade: cotidiano, saberes e práticas em saúde. Rio de Janeiro (RJ): IMS-UERJ/ABRASCO; 2003. p. 89-111.

13. Martins PH, Fontes B. Redes sociais e saúde: novas possibilidades teóricas. Recife (PE): Editora Universitária da UFPE; 2004

14. Secretaria Municipal de Saúde do Rio de Janeiro (BR). Regimento interno Parque Royal. Documento da unidade. Regimento interno CMS Parque Royal. Rio de Janeiro (RJ): Secretaria Municipal de Saúde do Rio de Janeiro; 2018.

15. Prefeitura do Rio de Janeiro (BR). Secretaria Municipal de Saúde do Rio de Janeiro. Saúde presente: o plano que está mudando a saúde do Rio. Rio de Janeiro (RJ): Prefeitura do Rio; 2019; [acesso em 2019 Jan 04]. Disponível em: http://www.rio.rj.gov.br/web/sms/ saude-presente

16. Borgatti SP. 2-Mode concepts in social network analysis. In: Meyers RA, ed. Encyclopedia of complexity and system science. Heidelberg: Springer; 2009.

17. Borgatti SP, Everett MG, Freeman LC. Ucinet for Windows: software for social network analysis. Harvard, MA: Analytic Technologies; 2002.

18. Conselho Nacional de Saúde (Brasil). Resolução no 466/2012 de 12 de dezembro de 2012. Aprova as seguintes diretrizes e normas regulamentadoras de pesquisas envolvendo seres humanos. Brasília, 2012; [acesso em 2019 jul 04]. Disponível em: https://conselho. saude.gov.br/resolucoes/2012/Reso466.pdf.

19. Raider H,Krackhardt DJ. Intraorganizational networks. In: Baum JAC, ed. Companion to organizations. Oxford: Blackwell Publishers; 2001. p. 58-74.

20. Chiara IGD. As citações como base da rede social egocêntrica: o artigo citado e suas conexões. Associação Nacional de Pesquisa Pós-Graduação em Ciência da Informação (ANCIB); 1-4 ago 2006; Londrina, PR, Brasil. Londrina (PR): ANCIB; 2006 ; [acesso em 2019 Fev 24]. Disponível em: http://enancib.ibict.br/index.php/enancib/viienancib/paper/viewFile/2470/1601 
21. Hatala JP. Social network analysis in human resource development: a new methodology. Human Resource Development Review. 2006 Mar;5(1):45-71. DOI: https://doi.org/10.1177/1534484305284318 DOI: https://doi.org/10.1177/1534484305284318

22. Carmo ME, Guizardi FL. O conceito de vulnerabilidade e seus sentidos para as políticas públicas de saúde e assistência social. Cad Saúde Pública. 2018;34(3):e00101417. DOI: http://dx.doi.org/10.1590/0102-311x00101417 DOI: https://doi.org/10.1590/0102-311x00101417

23. Merhy EE. Saúde: a cartografia do trabalho vivo. $3^{\text {a }}$ ed. São Paulo: Hucitec; 2002.

24. Silva DC, Alvim NAT, Figueiredo PA. Tecnologias leves em saúde e sua relação com o cuidado de enfermagem hospitalar. Esc Anna Nery. 2008;12(2):291-8.DOI: http://dx.doi.org/10.1590/S1414-81452008000200014 DOI: https://doi.org/10.1590/S1414-81452008000200014

25. Sousa FG, Terra MG, Erdmann AL. Health services organization according to the intersectoral perspective: a review. Online Braz J Nurs. 2005;4(3):1-4. DOI: https://doi.org/10.5935/1676-4285.200544

26. Braga F, Fazito D. Análise de redes sociais e as conexões territoriais da migração no Brasil: padrões estruturais da migração interna entre 1980 e 2000. In: Anais do XVII Encontro Nacional de Estudos Populacionais; 24 set 2010; Caxambú, MG, Brasil. Caxambú (MG): Associação Brasileira de Empresas de Pesquisa (ABEP); 2010. p. 1-21.

27. Morosini MV, Fonseca AF. Os agentes comunitários na Atenção Primária à Saúde no Brasil: inventário de conquistas e desafios. Saúde Debate.2018 Set; 42(spe1):261-74.DOI: http://dx.doi.org/10.1590/0103-11042018s117 DOI:https://doi.org/10.1590/0103-11042018s117

28. Bourdieu P. Meditações pascalianas. Rio de Janeiro: Bertrand Brasil; 2001.

29. Ministério da Saúde (BR). Acolhimento à demanda espontânea. Brasília (DF): Ministério da Saúde; 2011.

30. Conselho Federal de Enfermagem (COFEN). Resolução COFEN no 423/2012 de 11 de abril de 2012. Normatiza no Âmbito do Sistema Conselho Federal e Conselhos Regionais de Enfermagem a Participação do Enfermeiro na Atividade de Classificação de Riscos. Diário Oficial da União, Brasília, 2012; [acesso em 2019 set 16]. Disponível em: http://www.cofen.gov.br/resoluo-cofen-n-4232012_8956.html

31. Ginzburg C. Sinais: raízes de um paradigma indiciário. In: Mitos, emblemas, sinais: morfologia e história. 1a reimpr. São Paulo: Companhia das Letras; 1990.

32. Guimarães MBL. Intuição, pensamento e ação na clínica. Interface (Botucatu). 2005 Ago;9(17):317-32. DOI: http://dx.doi.org/10.1590/ S1414-32832005000200008 DOI: https://doi.org/10.1590/S1414-32832005000200008

33. Bourdieu P. Escritos de educação. Petrópolis: Vozes; 1998. 